\section{The SMAD2/3 corepressor SNON maintains pluripotency through selective repression of mesendodermal genes in human ES cells}

\author{
Norihiro Tsuneyoshi, ${ }^{1}$ Ee Kim Tan, ${ }^{1}$ \\ Akila Sadasivam, ${ }^{1}$ Yogavalli Poobalan, ${ }^{1}$ \\ Tomoyuki Sumi, ${ }^{2,3}$ Norio Nakatsuji, ${ }^{4,5}$ \\ Hirofumi Suemori, ${ }^{2}$ and N. Ray Dunn ${ }^{1,6}$ \\ ${ }^{1}$ Institute of Medical Biology, A*STAR (Agency for Science, \\ Technology, and Research), Singapore 138648, Singapore; \\ ${ }^{2}$ Department of Embryonic Stem Cell Research, Institute for \\ Frontier Medical Sciences, Kyoto University, Kyoto 606-8507, \\ Japan; ${ }^{3}$ Department of Developmental Biology, Graduate School \\ of Medical Sciences, Kyushu University, Fukuoka 812-8582, \\ Japan; ${ }^{4}$ Department of Development and Differentiation, \\ Institute for Frontier Medical Sciences, Kyoto University, Kyoto \\ 606-8507, Japan; ${ }^{5}$ Institute for Integrated Cell-Material Sciences \\ (WPI-iCeMS), Kyoto University, Kyoto 606-8501, Japan
}

Activin/Nodal signaling via SMAD2/3 maintains human embryonic stem cell (hESC) pluripotency by direct transcriptional regulation of $N A N O G$ or, alternatively, induces mesoderm and definitive endoderm (DE) formation. In search of an explanation for these contrasting effects, we focused on SNON (SKIL), a potent SMAD2/3 corepressor that is expressed in hESCs but rapidly down-regulated upon differentiation. We show that SNON predominantly associates with SMAD2 at the promoters of primitive streak (PS) and early DE marker genes. Knockdown of SNON results in premature activation of $P S$ and DE genes and loss of hESC morphology. In contrast, enforced SNON expression inhibits DE formation and diverts hESCs toward an extraembryonic fate. Thus, our findings provide novel mechanistic insight into how a single signaling pathway both regulates pluripotency and directs lineage commitment.

Supplemental material is available for this article.

Received July 23, 2012; revised version accepted September $28,2012$.

Since the isolation of human embryonic stem cells (hESCs) more than a decade ago, numerous extrinsic signals have been implicated in the maintenance of their cardinal features of pluripotency and indefinite selfrenewal (Pera and Tam 2010). These include growth factors belonging to the TGF $\beta$, FGF, WNT, and IGF families, which are often supplemented with culture

[Keywords: Activin/Nodal; SNON; human embryonic stem cells; mesendoderm; pluripotency; repressor]

${ }^{6}$ Corresponding author

E-mail ray.dunn@imb.a-star.edu.sg

Article is online at http://www.genesdev.org/cgi/doi/10.1101/gad.201772.112. medium containing serum, feeder cell-conditioned medium, and/or ill-defined knockout serum replacement (Price et al. 1998). These various and complex platforms have obscured the identification of the minimal complement of extracellular cues that govern the intrinsic transcriptional regulators of pluripotency and self-renewal. Recently, however, it was shown that either recombinant TGF $\beta 1$ or the related ligand Activin A in combination with FGF2 can maintain hESCs in chemically defined and feeder-free conditions partly through direct regulation of the core pluripotency factor gene NANOG (Xu et al. 2008; Vallier et al. 2009).

Both TGF $\beta$ and Activin signals are transduced intracellularly by phosphorylation of the cytoplasmic effector proteins SMAD2 and SMAD3 (Schmierer and Hill 2007). Once activated, they partner with SMAD4, translocate to the nucleus, and associate with tissue-specific transcription factors to regulate batteries of target genes. Chromatin immunoprecipitation (ChIP) experiments show that SMAD2/3 bind the NANOG promoter, and luciferase reporter assays confirm that this SMAD-binding element (SBE) is crucial for $N A N O G$ transcriptional activity $(\mathrm{Xu}$ et al. 2008; Vallier et al. 2009). Recently, SMAD2/3 ChIP combined with next-generation sequencing (ChIP-seq) revealed that, in addition to $N A N O G$, SMAD2/3 occupy a broad number of genes both expressed in hESCs and associated with the maintenance of pluripotency (Brown et al. 2011; Kim et al. 2011). These include the additional core pluripotency factors OCT4 and $S O X 2$ as well as other key regulators, such as SUZ12, UTF1, TERT, DPPA4, and $L I N 28 A$. Taken together, these findings emphasize the importance of continuous TGF $\beta /$ Activin signaling for the maintenance of hESC pluripotency. However, it is a third divergent TGF $\beta$-related ligand, Nodal, that carries out this fundamental activity in vivo.

Nodal transcripts identify the pluripotent cells within the mouse blastocyst inner cell mass (ICM) and persist in these cells as they extensively reorganize into the cupshaped epiblast epithelium after implantation (Mesnard et al. 2006; Arnold and Robertson 2009). In the absence of Nodal, null mutant embryos down-regulate Oct4, Nanog, and Foxd3, which are normally uniformly expressed throughout the epiblast; prematurely and ectopically upregulate neural markers; and fail to gastrulate (Camus et al. 2006; Mesnard et al. 2006). With the onset of gastrulation, Nodal expression resolves to the posterior primitive streak (PS), where epiblast cells ingress and emerge as mesoderm and definitive endoderm (DE) (Arnold and Robertson 2009). Extensive genetic studies have shown that the highest levels of Nodal specify DE, while the assorted mesodermal lineages are patterned by intermediate to lower Nodal levels (Arnold and Robertson 2009). Thus, there is a remarkable conversion in a narrow developmental time span in how Nodal signals are interpreted intracellularly-from maintenance of pluripotency within the ICM/epiblast to inducer of differentiation within the PS. This raises the intriguing question of how Nodal/Smad2/3 signals in the ICM/epiblast engage only the pluripotency machinery and avert precocious activation of differentiation genes. This question also stringently applies to hESCs, which share many defining characteristics with the mouse epiblast and its derivative cell lines (termed epiblast stem cells [EpiSCs]) (Pera and Tam 2010), 
as the Nodal analog Activin A both regulates pluripotency and is nearly universally used to initiate mesendoderm differentiation in vitro to produce a variety of lineagespecific cell types (Sulzbacher et al. 2009).

We reasoned that one explanation for these contrasting effects is the existence of discriminating nuclear repressor proteins. Moreover, the expression of such a repressor would likely be under the direct control of the core pluripotency factors. Previous ChIP combined with DNA microarrays (ChIP-chip) identified numerous active and inactive genes that are triply bound by OCT4, SOX2, and NANOG in hESCs (Boyer et al. 2005). One of these is the SKI-related proto-oncogene SNON (SKIL) that encodes a potent negative regulator of TGF $\beta$-related signaling (Deheuninck and Luo 2009; Zhu and Luo 2012). SNON physically interacts with SMAD2, SMAD3, and SMAD4, disrupting their assembly into an activated heteromeric complex (Stroschein et al. 1999; Wu et al. 2002). In addition, SNON prevents SMAD2 and SMAD3 from associating with the transcriptional coactivator p300/CBP and recruits a repressor complex comprised of nuclear receptor corepressor (N-CoR) and histone deacetylase (HDAC) (Schmierer and Hill 2007). Thus, the presence of SNON at a particular SBE ensures transcriptional repression. Here, we found that SNON protein is highly abundant in hESCs and specifically enriched at differentiation gene promoters. Loss of SNON in hESCs prematurely activates PS and early DE genes and destabilizes pluripotency, while constitutive SNON expression restrains differentiation into DE. Taken together, our results identify SNON as a key component of the nuclear machinery that safeguards pluripotency through direct repression of differentiation genes downstream from Activin/Nodal.

\section{Results and Discussion}

SNON is abundant in hESCs and is targeted to the proteasome during early differentiation

We first sought to confirm the expression of $S N O N$ in pluripotent hESCs and examine its regulation during DE formation in vitro. Differentiation was carried out according to the schematic in Figure 1A (Teo et al. 2012) and, expectedly, resulted in the loss of pluripotency markers (NANOG, OCT4, and SOX2) and up-regulation of PS (WNT3, FGF8, EOMES, and MIXL1), mesendoderm (GSC and $L H X 1)$, and DE (FOXA2 and SOX17) markers by day 5 (Supplemental Fig. 1A). These data were confirmed by Western analysis, immunohistochemistry, and FACS (Supplemental Fig. 1B-D). We observed that SNON transcript levels are robust in undifferentiated HES3 and on day 1 of differentiation but drop considerably by day 3 and decline further on day 5 (Fig. 1B).

This decrease in SNON levels in vitro closely mirrors the expression dynamics of $S n o N$ in the early mouse embryo. Prior to gastrulation, SnoN transcripts label the pluripotent epiblast epithelium (Supplemental Fig. 1E). Mouse ESCs and EpiSCs also express SnoN (Supplemental Fig. 1F). With the onset of gastrulation, SnoN transcript levels are conspicuously down-regulated in the region of the incipient PS and continue to decline posteriorly as the PS lengthens (Supplemental Fig. 1E). Nascent mesoderm and DE also lack SnoN expression. By the late PS stage (embryonic day 7.5 [E7.5]), SnoN transcripts resolve to the anterior epiblast, but shortly
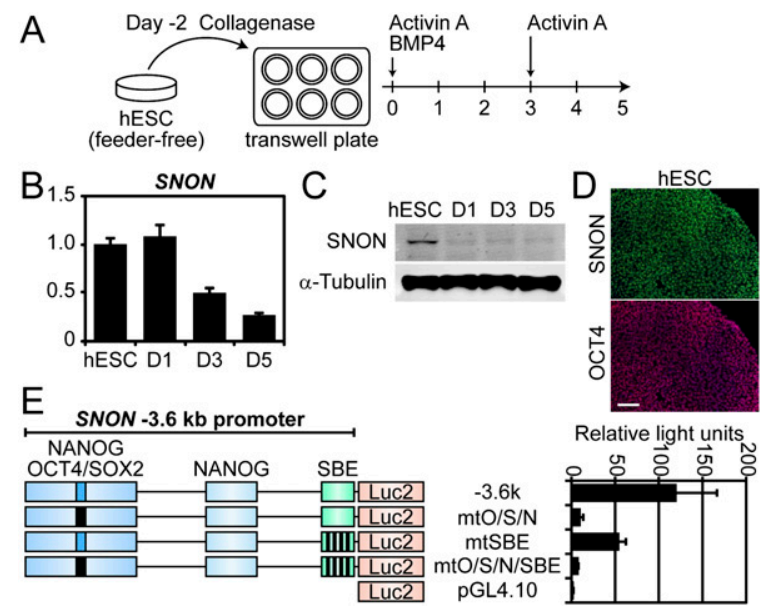

Figure 1. SNON expression in hESCs is controlled by SMAD2/3 and OCT4, SOX2, and NANOG. $(A)$ hESCs were differentiated into DE for $5 \mathrm{~d}$ according to the schematic. Activin A and BMP4 (both at $50 \mathrm{ng} / \mathrm{mL}$ ) were added on day 0. Only Activin A was replenished on day 3. (B) SNON expression levels during DE differentiation by qPCR. Data were normalized against GUSB and are shown relative to undifferentiated hESCs $(=1.0) .(C)$ Western blot analysis for SNON protein levels during DE differentiation. (D) OCT4 and SNON immunofluorescence on undifferentiated HES3 (hESCs). Bar, $100 \mu \mathrm{m}$. (E) Luciferase reporter assays of SNON promoter activity in HES3. Black shading indicates specific mutation of the OCT4/SOX2/NANOG-binding sites in the $5^{\prime}$ distal enhancer or of the four SBEs proximal to the SNON transcriptional start site.

thereafter, this domain has largely disappeared. These expression data suggest that loss of the Smad2/3 corepressor SnoN is an obligate step in the production of PS-derived lineages.

At the protein level, SNON is abundant in undifferentiated HES3 but, in contrast to our quantitative PCR (qPCR) data, drastically reduced by day 1 (Fig. 1C,D). SKI, which is closely related to SNON, is also present in hESCs, but its levels fluctuate negligibly between days 1 and 5 (Supplemental Fig. 1B,G). Several E3 ubiquitin ligases previously shown to target $\mathrm{SNON}$ to the proteasome are expressed in hESCs and during differentiation, including SMURF2, the RING domain-containing protein ARKADIA (RNF111), and the anaphase-promoting complex/cyclosome (APC/C) (Deheuninck and Luo 2009). SMURF2 levels are up-regulated on day 1 and then plateau, while expression of $A R K A D I A$ and two APC/C subunits-CDH1 (FZR1) and ANAPC2-increase gradually (Supplemental Fig. 1G). Similar results were observed by Western for SMURF2 and ARKADIA (Supplemental Fig. 1B). Other APC/C components, including ANAPC11, $C D C 16$, and $C D C 27$, were equally expressed at all time points (Supplemental Fig. 1G).

To confirm that the rapid loss of SNON protein is indeed due to proteasomal degradation, feeder-free HES3 cells were treated with two proteasome inhibitors: MG132 and lactacystin. When HES3 cells are differentiated in the presence of MG132 or lactacystin, SNON protein levels expectedly persist on day 1 (Supplemental Fig. $1 \mathrm{H}$ ). Taken together, these data show that $S N O N$ expression is tightly linked to pluripotency and that SNON is targeted to the proteasome at the onset of DE formation. 
SNON expression in hESCs is controlled by $S M A D 2 / 3$ and OCT4, SOX2, and NANOG

ChIP-chip, recent ChIP-seq, and phylogenetic alignments reveal a series of conserved SMAD2/3- and OCT4/SOX2/ NANOG-binding regions within the $\sim 5-\mathrm{kb} S N O N$ promoter/enhancer (Fig. 1E; Supplemental Fig. 1I; Boyer et al. 2005; Zhu et al. 2005; Brown et al. 2011; Kim et al. 2011). We investigated the contribution of each of these elements to SNON regulation in undifferentiated hESCs using luciferase reporter constructs. Serial deletion analysis showed that the distal 5' OCT4/SOX2/NANOGbinding region is essential for robust reporter activity (Supplemental Fig. 1I). The more proximal NANOG and SMAD2/3 elements contribute modestly to the regulation of SNON when compared with the $-3.6-\mathrm{kb}$ construct (Supplemental Fig. 1I). We also generated a series of -3.6-kb reporters in which OCT4/SOX2/NANOGand SMAD2/3-binding sites were individually mutated (Fig. 1E; Supplemental Table 1). Consistent with our deletion studies, the mutated OCT4/SOX2/NANOG $(\mathrm{mtO} /$ $\mathrm{S} / \mathrm{N}$ ) construct in which distal OCT4/SOX2/NANOG binding is specifically disrupted shows significantly reduced ( $\sim 13$-fold) reporter activity (Fig. 1E). In contrast, mutating all four SBEs (mtSBEs) diminishes SNON reporter activity roughly twofold (Fig. 1E). Taken together, these data establish that $S N O N$ is TGF $\beta$-responsive and that OCT4/SOX2/NANOG serve as critical upstream regulators of $S N O N$ expression in hESCs.

\section{SNON knockdown destabilizes pluripotency}

To analyze SNON function, we first performed tetracycline (Tet)-inducible shRNA-mediated knockdown of SNON in S4TR5 cells, a derivative of the Shef4 hESC line that constitutively expresses the nuclear Tet repressor (TetRnls) (Zafarana et al. 2009). Two independent and previously validated human SNON-shRNA target sequences-shSNON(954) and shSNON(1307)were transfected into S4TR5 cells (Sarker et al. 2005; $\mathrm{Zhu}$ et al. 2007). After selection, 11 of 12 clones for $\operatorname{shSNON}(954)$ and 10 of 12 clones for $\operatorname{shSNON(1307)}$ showed $\sim 80 \%$ SNON knockdown by qPCR after $3 \mathrm{~d}$ of doxycycline (Dox) treatment (data not shown). Two

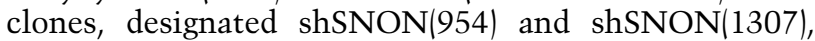
were chosen for further study. Both were indistinguishable by morphology (Supplemental Fig. 2A) and showed OCT4, SOX2, and NANOG levels comparable with the parental S4TR5 line by qPCR and Western (Supplemental Fig. 2B,C). Dox treatment resulted in a significant knockdown of $S N O N$ transcripts on day 1 (Fig. 2A,B). Interestingly, both shSNON clones lost their compact colony morphology after $5 \mathrm{~d}$ of continuous culture in Dox and appeared differentiated (Supplemental Fig. 2A). This was accompanied by a specific increase in PS (BRACHYURY, $M I X L 1$, and $E O M E S)$, mesendoderm (GSC), and early DE (FOXA2 and SOX17) markers beginning around day 3 (Fig. 2B; Supplemental Fig. 2B). Neuroectodermal gene expression was not above background (Supplemental Fig. 2B) and, importantly, levels of OCT4, SOX2, and NANOG remained unchanged (Supplemental Fig. 2B,C). In addition, loss of SNON did not impact SKI, SMURF2, SMAD2/pSMAD2, or SMAD3/pSMAD3 levels, suggesting that the genes encoding these proteins are not regulated by the SNON corepressor in hESCs (Supplemental Fig. 2C). Consistent with our qPCR data, Western analysis
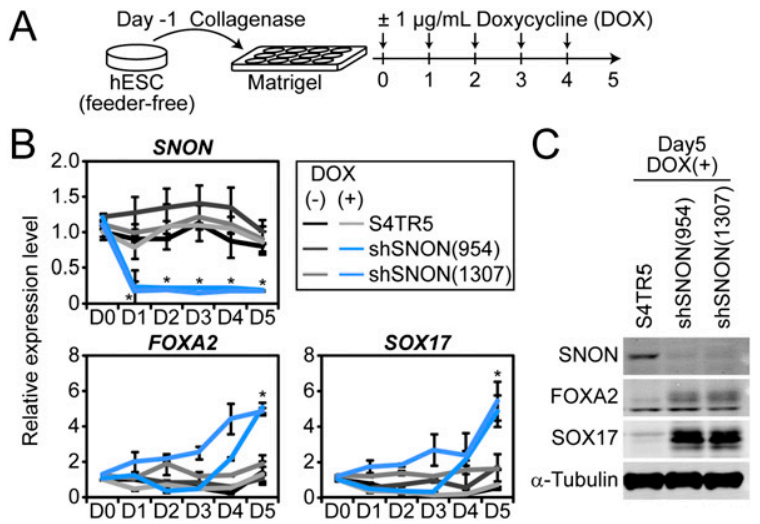

Figure 2. SNON knockdown induces differentiation in hESCs. $(A)$ Schematic of the experimental design using two stable DOX-inducible S4TR5 hESC lines-shSNON(954) and shSNON(1307)-where DOX was replenished daily for 5 d. (B) SNON, FOXA2, and SOX17 expression analysis by qPCR. RNA was collected on days 0-5 according to $A$. Data were normalized against GUSB and are depicted relative to S4TR5 (DOX - ) at day $0(=1.0)$. $P$-values were calculated according to the Student's $t$-test. $\left(^{\star}\right) P<0.05$. (C) Western blot analysis for SNON, FOXA2, and SOX17.

revealed that SNON is hardly detectable on day 1 , and low levels of FOXA2 were first detected on day 2, followed by SOX17 on day 5 (Fig. 2C; Supplemental Fig. 2D). Immunofluorescence also confirmed loss of SNON, persistence of OCT4, and up-regulation of SOX17 and FOXA2 on day 5 (Supplemental Fig. 2E,F).

To provide additional evidence that loss of SNON results in differentiation, we performed a replating experiment whereby S4TR5 cells and both knockdown clones were passaged after $5 \mathrm{~d}$ of Dox treatment and cultured for four additional days in Dox. OCT4-DAB staining was used to identify hESC colonies and clearly revealed the dramatically reduced plating efficiency of the shSNON knockdown clones after passaging (Supplemental Fig. 2G). Taken together, our results show that diminished SNON levels in hESCs destabilizes pluripotency through precocious activation of mesendodermal genes.

\section{SNON overexpression blocks differentiation into $D E$ and mesoderm}

We next performed the reciprocal experiment by overexpressing SNON in hESCs. After repeated attempts, we were unable to isolate HES3 subclones with levels greater than endogenous SNON (data not shown). We therefore chose to overexpress a stable truncated form of SNONSNON(1-366) - that retains its ability to bind SMAD2/3 and is sufficient for transcriptional repression but lacks the C-terminal lysine resides critical for ubiquitination and proteasomal degredation (Stroschein et al. 1999, 2001). Two SNON(1-366) clones (\#1 and \#9) were isolated that produce roughly equivalent levels of SNON(1-366) (Fig. 3A) and remain undifferentiated, with OCT4 immunoreactivity comparable with wild-type HES3 (Supplemental Fig. 3A).

Clones \#1 and \#9 were then subjected to DE differentiation according to the schematic in Figure 1A, along with two controls: wild-type HES3 and HES3 harboring pCAG-IRES-puro (vector control). NANOG, OCT4, SOX2, and DPPA4 levels declined with similar kinetics in controls 


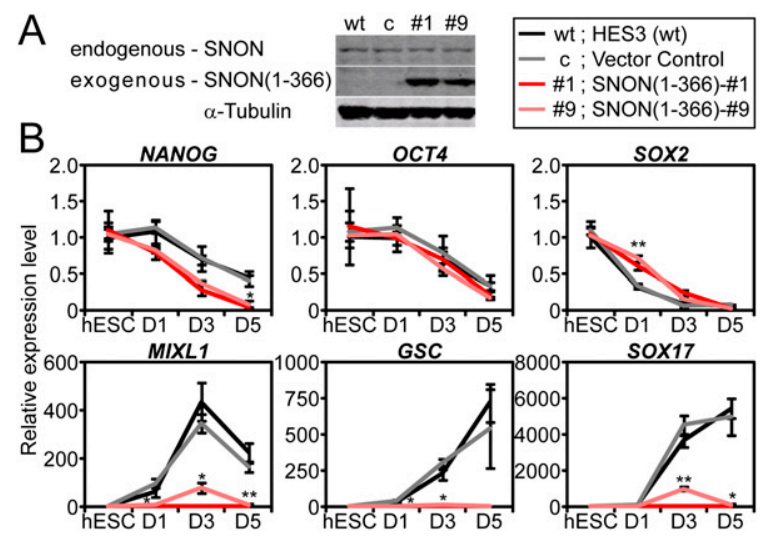

Figure 3. SNON overexpression inhibits DE formation. $(A)$ Western blot analysis detects abundant C-terminally truncated, constitutively active SNON(1-366) in two stable, clonally selected lines (\#1 and \#9). (B) Expression analysis of the indicated genes by qPCR. hESCs were differentiated according to the schematic in Figure 1A. Data were normalized against GUSB and are shown relative to parental wild-type undifferentiated hESCs (= 1.0). The "vector control" HES3 line contains only the pCAG-IRES-Puro construct. $P$-values were calculated according to the Student's $t$-test. $\left(^{\star}\right) P<$ $\left.0.05 ;{ }^{\star \star}\right) P<0.01$.

and clones \#1 and \#9 (Fig. 3B; Supplemental Fig. 3C). HES3 and the vector control cell line predictably showed up-regulation of the PS markers (BRACHYURY, WNT3, FGF8, MIXL1, and EOMES) as well as mesendodermal (LHX1 and GSC) and DE (FOXA2 and SOX17) markers beginning on day 1 (Fig. 3B; Supplemental Fig. 3C). Clones $\# 1$ and \#9 failed to activate the expression of PS, mesendodermal, and DE marker genes, all of which are known Activin/Nodal targets and bound by SMAD2/3 (Fig. 3B; Supplemental Fig. 3C; Schmierer and Hill 2007; Arnold and Robertson 2009). This result is entirely consistent with the ability of SNON(1-366) to strongly suppress the synthetic ARE-lux Activin/Nodal pathway reporter, which contains three repeats of a Xenopus Activin response element (ARE) that binds SMAD2/4 and the forkhead transcription factor FOXH1. Human GSC-Luc2 and MIXL1-Luc2 reporters containing their respective endogenous AREs are similarly repressed by SNON(1-366) (Supplemental Fig. 3D). When confronted with culture conditions tailored to produce cardiomyocytes (Hudson et al. 2012), SNON(1-366)-overexpressing clones also fail to up-regulate early mesodermal progenitor markers (Supplemental Fig. 3E). SNON(1-366) therefore serves as a potent inhibitor of both mesoderm and DE formation downstream from Activin/Nodal signals. Consequently, SNON(1-366)-expressing clones initiate extraembryonic lineage differentiation in response to Activin A and BMP4 treatment, as evidenced by the up-regulation of the trophoblast marker genes CDX2, CGA, CYP19A, GCM1, KRT7, $L G A L S 16$, and VGLL1 and the visceral/parietal endoderm marker SOX7 (Supplemental Fig. 3C). In addition, no induction of early neuroectodermal markers (NEUROD1 and SOX1) was observed (Supplemental Fig. 3C).

\section{SNON occupies SBEs in mesendodermal genes}

To formally test whether SNON binds and represses PS and mesendodermal genes in undifferentiated hESCs, we performed SMAD2, SMAD3, and SNON ChIP followed by qPCR for enrichment at select SBEs. Given the high percentage of amino acid identity between SMAD2 and SMAD3 and the potential for antibody cross-reactivity, we used monoclonal anti-SMAD2 and anti-SMAD3 antibodies that specifically distinguish between these two proteins (Supplemental Figs. 1K, 4B). Among "stem cell factor" genes (NANOG, OCT4, SOX2, DPPA4, and LIN28) that are highly expressed in hESCs, SNON occupancy at their respective SBEs was low to undetectable (Fig. 4A; Supplemental Fig. 4A). Only SUZ12 showed statistically significant enrichment for SNON $(P<0.05)$. However, SUZ12, like the other stem cell factor genes analyzed, was insensitive to SNON(1-366) overexpression (Supplemental Fig. 3C). In contrast, SNON highly occupied PS (FGF8, WNT3, EOMES, and MIXL1) and mesendodermal (GSC and LHX1) genes (Fig. 4A; Supplemental Fig. 4A). SMAD2 bound all SBEs analyzed by ChIP (Fig. 4A; Supplemental Fig. 4A). Consistent with the ability of SNON to recruit HDACs, acetylated H3 and $\mathrm{H} 4$ levels at PS/mesendodermal genes were low in undifferentiated HES3 cells but increased with differentiation (Supplemental Fig. 4C; Xi et al. 2011). The converse was observed at stem cell factor gene promoters, with declining acetylation mirroring the decrease in pluripotency gene expression during differentiation (Supplemental Figs. 1A, 3C, 4C). Taken together, these data are wholly consistent with SNON in combination with SMAD2/SMAD4 acting as a key transcriptional repressor of differentiation-specific genes in pluripotential hESCs (Fig. 4B).

Our ChIP data also reveal a bias of SMAD3 toward stem cell factor gene promoters (Fig. 4A; Supplemental Fig. 4A). In a very recent study, Mullen et al. (2011) reported that OCT4 and SMAD3 are predominantly associated with transcriptionally active genes in hESCs and, importantly, co-occupy the genome by binding adjacent DNA sites. Our results show that no repression of pluripotency genes was observed in hESC clones overexpressing SNON(1-366), which retains its ability to bind SMAD3 but cannot be targeted by E3 ligases (Fig. 3B; Supplemental Fig. 3C). These genes include $N A N O G$, whose regulation by TGF $\beta /$ Activin/SMAD2/3 in hESCs is extremely well characterized (Xu et al. 2008; Vallier et al. 2009). If SNON were a general repressor downstream from TGF $\beta$-related signaling, modulating the overall activity of the TGF $\beta$ pathway in hESCs, ectopic expression of SNON(1-366) would be predicted to suppress NANOG and other crucial pluripotency genes and promote differentiation. This is not what we observed (Fig. 3B; Supplemental Fig. 3D). We therefore propose that OCT4 serves a central "protective" role in averting SNON repression of SMAD3-bound pluripotency genes (Fig. 4B). In contrast, selective recruitment of SNON represses PS/mesendoderm genes in hESCs until extrinsic cues trigger its degradation and allow differentiation to unfurl.

One outstanding question is thus the identity of the E3 ligases specifically responsible for the rapid clearance of SNON at the onset of differentiation. Genetic evidence in mice favors a role for Arkadia because Arkadia-null mutant embryos succumb to severe gastrulation defects due to impaired Nodal/Smad2/3 signaling (Episkopou et al. 2001; Mavrakis et al. 2007). One interpretation of this phenotype is that in the absence of Arkadia, SnoN associates with crucial Nodal/Smad2/3 target genes, 
A

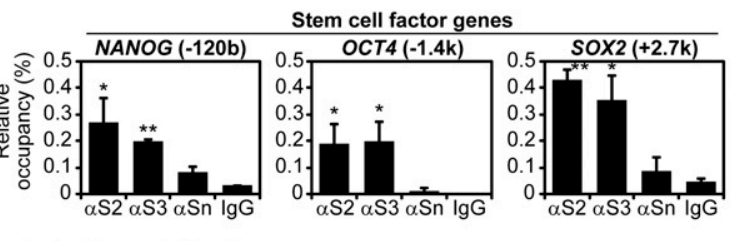

Stem cell factor genes

Pluripotent human ES cells
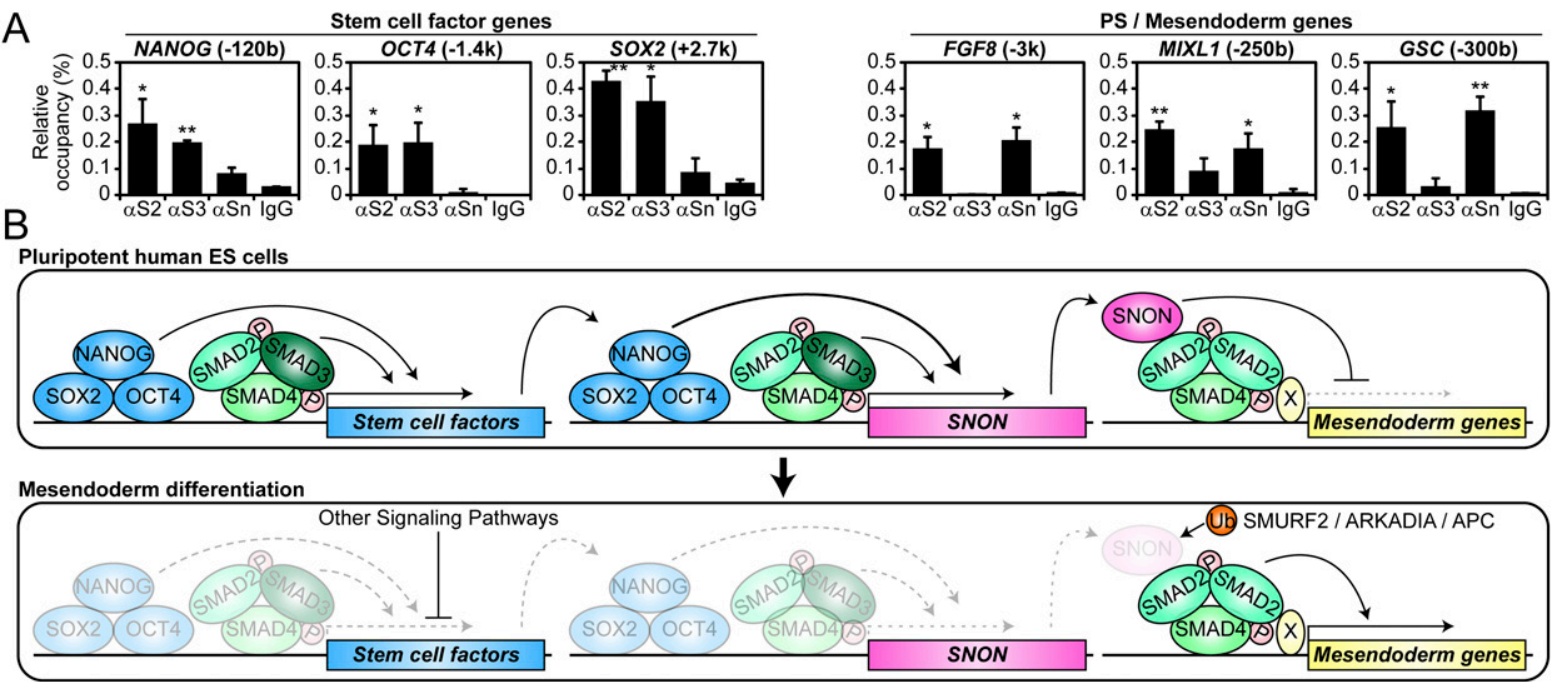

Figure 4. SNON highly and selectively occupies the promoters of PS and mesendodermal genes in hESCs. $(A)$ ChIP-qPCR analysis of SNONbinding $(\alpha S n)$, SMAD2-binding $(\alpha S 2)$, and SMAD3-binding $(\alpha S 3)$ sites at the indicated gene regions in HES3 cultured in mTeSR1 medium. Relative occupancy values are shown as the apparent immunoprecipitation efficiency (percentage) (ratio $=$ immunoprecipitated DNA/input DNA). (IgG) Normal rabbit IgG as negative control. $P$-values were calculated according to the Student's $t$-test. $\left({ }^{\star}\right) P<0.05$; $\left(^{\star \star}\right) P<0.01$. $(B)$ Proposed model for SNON function in hESCs. In pluripotent hESCs, extrinsic signals (e.g., TGF $\beta /$ Activin/Nodal) regulate stem cell factor gene expression through activated SMAD2/3/4 complexes. OCT4/SOX2/NANOG positively regulate their own promoters and activate $S N O N$ transcription. SMAD2/3 also regulate SNON. SNON is selectively recruited to SMAD2-bound mesendodermal genes and suppresses their transcription. During early differentiation, stem cell factor gene expression and SMAD2/3/4 occupancy at the SNON promoter/enhancer decline. Consequently, SNON expression decreases. Existing SNON protein is rapidly degraded in a ligand-dependent manner by E3 ubiquitin ligases (e.g., SMURF2, ARKADIA, or APC). This leads to the derepression of early PS and DE target genes (collectively referred to as mesendoderm). (X) Tissue-specific transcriptional coactivators such as FOXH1.

preventing their activation. Consistent with this prediction, SnoN protein accumulates in Arkadia-deficient mouse ESC lines (Nagano et al. 2007). Thus, SNON degradation in hESCs may similarly require the stimulus-dependent activation of ARKADIA or the synergistic involvement of other ligand-dependent E3 ligases, such as SMURF2 (Bonni et al. 2001). It is important to note that there are $>600$ E3 ligases and substrate recognition subunits encoded by the human genome (Li et al. 2008), many of which are enriched in hESCs (Assou et al. 2009). This finding presages the heightened sensitivity that hESCs exhibit to proteasome inhibitors (Assou et al. 2009; Vilchez et al. 2012; data not shown) and further suggests that there are additional hitherto uncharacterized regulators that act alongside ARKADIA, APC, and SMURF2 to target SNON to the proteasome.

\section{Materials and methods}

\section{Cell culture}

HES3 cells were maintained as previously described (Suemori et al. 2006). For feeder-free culture, HES3 and S4TR5 cells (Zafarana et al. 2009) were maintained in mTeSR1 medium (Stem Cell Technologies) on 1:200 GFRMatrigel-coated (Becton Dickinson) dishes. DE differentiation was carried out as previously described (Teo et al. 2012).

\section{Tet-inducible shRNA knockdown}

Previously validated human SNON shRNA target sequences were ligated into pSUPERIOR-Zeocin (Supplemental Material; Sarker et al. 2005; Zhu et al. 2007). S4TR5 cells were transfected with the shRNA constructs using FuGene HD (Roche) and selected for 2 wk using $2.5 \mu \mathrm{g} / \mathrm{mL}$ Zeocin (Invitrogen).

\section{SNON overexpression}

Human $S N O N(1-366)$ cDNA was PCR-amplified and ligated into pCAGIRES-Puro (Supplemental Material; Sumi et al. 2007). Feeder-free HES3 cells were transfected with pCAG-SNON(1-366)-IRES-Puro or empty vector using FuGene HD and selected for 2 wk using $1 \mu \mathrm{g} / \mathrm{mL}$ puromycin (Sigma).

\section{qPCR analysis}

Total RNA was extracted using the RNeasy kit (Qiagen), and 0.5-2 $\mu$ g was reverse-transcribed with the High-Capacity cDNA Reverse Transcription kit (Applied Biosystems). Real-time PCR was performed using TaqMan Fast Advanced Master Mix or Power SYBR Green PCR Master Mix. Relative quantitation was performed using $2^{-\Delta \Delta C \mathrm{CT}}$ and normalized against GUSB. TaqMan gene expression assays and primers are listed in Supplemental Table 2.

\section{Luciferase assay}

An $\sim 5$-kb segment of the human SNON promoter was PCR-amplified (Supplemental Table 1) and subcloned into pGL4.10[luc2] (Promega). pTKhRluc was used for data normalization (Supplemental Material). Molar equivalents of each luc2 reporter vector and $0.05 \mu \mathrm{g}$ of pTK-hRluc were cotransfected into HES3 using FuGene HD. After 48 h, both firefly and Renilla luciferase activities were measured using the Dual-Luciferase Reporter Assay system (Promega). Relative luciferase units were calculated by determining the ratio between firefly and Renilla luciferase activities and normalizing against the pGL4.10 vector control.

\section{Immunofluorescence and immunocytochemical analysis}

For immunofluorescence, hESCs were fixed in $3.7 \%$ formaldehyde/PBS for $15 \mathrm{~min}$, permeabilized with $0.2 \%$ Triton X-100/PBS for $5 \mathrm{~min}$, and incubated with primary antibodies (Supplemental Table 3). After incubation with Alexa Fluor 488- or 594-conjugated secondary antibodies 
(Invitrogen), nuclei were counterstained with DAPI (Sigma). For immunocytochemical staining of OCT4, hESCs were fixed, permeabilized, and incubated with anti-OCT4 antibody (Supplemental Table 3). After incubation with HRP-conjugated secondary antibody (Dako), antigens were visualized with DAB (Sigma).

\section{Western blot analysis}

Cell lysates were separated by SDS-PAGE, transferred to Immobilon-FL PVDF membrane (Millipore), and probed with primary antibodies (Supplemental Table 3). After incubation with IRDye 800CW- or 680LTconjugated secondary antibodies (LI-COR), proteins were detected using the Odyssey infrared imaging system (LI-COR).

\section{ChIP and quantitative real-time PCR}

ChIP was performed as described previously (Boyer et al. 2005). Additional details are provided in the Supplemental Material.

\section{Acknowledgments}

We thank Stuart Avery and Peter Andrews for providing S4RT5 cells, Ludovic Vallier for mEpiSC RNA, and Alan Colman, Dmitry Bulavin, Leah Vardy, Matthew Lovatt, Oz Pomp, Elizabeth Robertson, Elizabeth Bikoff, and Oliver Dreesen for insightful discussions and comments on the manuscript.

\section{References}

Arnold SJ, Robertson EJ. 2009. Making a commitment: Cell lineage allocation and axis patterning in the early mouse embryo. Nat ReV Mol Cell Biol 10: 91-103.

Assou S, Cerecedo D, Tondeur S, Pantesco V, Hovatta O, Klein B, Hamamah S, De Vos J. 2009. A gene expression signature shared by human mature oocytes and embryonic stem cells. BMC Genomics 10: 10. doi: 10.1186/1471-2164-10-10.

Bonni S, Wang HR, Causing CG, Kavsak P, Stroschein SL, Luo K, Wrana JL. 2001. TGF- $\beta$ induces assembly of a Smad2-Smurf2 ubiquitin ligase complex that targets SnoN for degradation. Nat Cell Biol 3: 587-595.

Boyer LA, Lee TI, Cole MF, Johnstone SE, Levine SS, Zucker JP, Guenther MG, Kumar RM, Murray HL, Jenner RG, et al. 2005. Core transcriptional regulatory circuitry in human embryonic stem cells. Cell 122: 947-956.

Brown S, Teo A, Pauklin S, Hannan N, Cho CH, Lim B, Vardy L, Dunn NR, Trotter M, Pedersen R, et al. 2011. Activin/Nodal signaling controls divergent transcriptional networks in human embryonic stem cells and in endoderm progenitors. Stem Cells 29: 1176-1185.

Camus A, Perea-Gomez A, Moreau A, Collignon J. 2006. Absence of Nodal signaling promotes precocious neural differentiation in the mouse embryo. Dev Biol 295: 743-755.

Deheuninck J, Luo K. 2009. Ski and SnoN, potent negative regulators of TGF- $\beta$ signaling. Cell Res 19: 47-57.

Episkopou V, Arkell R, Timmons PM, Walsh JJ, Andrew RL, Swan D. 2001. Induction of the mammalian node requires Arkadia function in the extraembryonic lineages. Nature 410: 825-830.

Hudson J, Titmarsh D, Hidalgo A, Wolvetang E, Cooper-White J. 2012. Primitive cardiac cells from human embryonic stem cells. Stem Cells Dev 21: 1513-1523.

Kim SW, Yoon SJ, Chuong E, Oyolu C, Wills AE, Gupta R, Baker J. 2011. Chromatin and transcriptional signatures for Nodal signaling during endoderm formation in hESCs. Dev Biol 357: 492-504.

Li W, Bengtson MH, Ulbrich A, Matsuda A, Reddy VA, Orth A, Chanda SK, Batalov S, Joazeiro CA. 2008. Genome-wide and functional annotation of human E3 ubiquitin ligases identifies MULAN, a mitochondrial E3 that regulates the organelle's dynamics and signaling. PLOS ONE 3: e1487. doi: 10.1371/journal.pone.0001487.

Mavrakis KJ, Andrew RL, Lee KL, Petropoulou C, Dixon JE, Navaratnam N, Norris DP, Episkopou V. 2007. Arkadia enhances Nodal/TGF- $\beta$ signaling by coupling phospho-Smad2/3 activity and turnover. PLoS Biol 5: e67. doi: 10.1371/journal.pbio.0050067.

Mesnard D, Guzman-Ayala M, Constam DB. 2006. Nodal specifies embryonic visceral endoderm and sustains pluripotent cells in the epiblast before overt axial patterning. Development 133: 24972505.

Mullen AC, Orlando DA, Newman JJ, Lovén J, Kumar RM, Bilodeau S, Reddy J, Guenther MG, DeKoter RP, Young RA. 2011. Master transcription factors determine cell-type-specific responses to TGF$\beta$ signaling. Cell 147: 565-576.

Nagano Y, Mavrakis KJ, Lee KL, Fujii T, Koinuma D, Sase H, Yuki K, Isogaya K, Saitoh M, Imamura T, et al. 2007. Arkadia induces degradation of SnoN and c-Ski to enhance transforming growth factor- $\beta$ signaling. I Biol Chem 282: 20492-20501.

Pera MF, Tam PP. 2010. Extrinsic regulation of pluripotent stem cells. Nature 465: 713-720.

Price PJ, Goldsborough MD, Tilkins ML. 1998. Embryonic stem cell serum replacement. International Patent Application WO 98/30679.

Sarker KP, Wilson SM, Bonni S. 2005. SnoN is a cell type-specific mediator of transforming growth factor- $\beta$ responses. I Biol Chem 280: 13037-13046.

Schmierer B, Hill CS. 2007. TGFß-SMAD signal transduction: Molecular specificity and functional flexibility. Nat Rev Mol Cell Biol 8: 970982.

Stroschein SL, Wang W, Zhou S, Zhou Q, Luo K. 1999. Negative feedback regulation of TGF- $\beta$ signaling by the SnoN oncoprotein. Science 286: 771-774.

Stroschein SL, Bonni S, Wrana JL, Luo K. 2001. Smad3 recruits the anaphase-promoting complex for ubiquitination and degradation of SnoN. Genes Dev 15: 2822-2836.

Suemori H, Yasuchika K, Hasegawa K, Fujioka T, Tsuneyoshi N, Nakatsuji N. 2006. Efficient establishment of human embryonic stem cell lines and long-term maintenance with stable karyotype by enzymatic bulk passage. Biochem Biophys Res Commun 345: 926-932.

Sulzbacher S, Schroeder IS, Truong TT, Wobus AM. 2009. Activin A-induced differentiation of embryonic stem cells into endoderm and pancreatic progenitors-the influence of differentiation factors and culture conditions. Stem Cell Rev 5: 159-173.

Sumi T, Tsuneyoshi N, Nakatsuji N, Suemori H. 2007. Apoptosis and differentiation of human embryonic stem cells induced by sustained activation of c-Myc. Oncogene 26: 5564-5576.

Teo AKK, Ali Y, Wong KY, Chipperfield H, Sadasivam A, Poobalan Y, Tan EK, Wang ST, Abraham S, Tsuneyoshi N, et al. 2012. Activin and BMP4 synergistically promote formation of definitive endoderm in human embryonic stem cells. Stem Cells 30: 631-642.

Vallier L, Mendjan S, Brown S, Chng Z, Teo A, Smithers LE, Trotter MW, Cho CH, Martinez A, Rugg-Gunn P, et al. 2009. Activin/Nodal signalling maintains pluripotency by controlling Nanog expression. Development 136: 1339-1349.

Vilchez D, Boyer L, Morantte I, Lutz M, Merkwirth C, Joyce D, Spencer B, Page L, Masliah E, Berggren WT, et al. 2012. Increased proteasome activity in human embryonic stem cells is regulated by PSMD11. Nature 489: 304-308.

Wu JW, Krawitz AR, Chai J, Li W, Zhang F, Luo K, Shi Y. 2002. Structural mechanism of Smad4 recognition by the nuclear oncoprotein Ski: Insights on Ski-mediated repression of TGF- $\beta$ signaling. Cell 111: 357-367.

Xi Q, Wang Z, Zaromytidou AI, Zhang XH, Chow-Tsang LF, Liu JX, Kim H, Barlas A, Manova-Todorova K, Kaartinen V, et al. 2011. A poised chromatin platform for TGF- $\beta$ access to master regulators. Cell 147: 1511-1524.

Xu RH, Sampsell-Barron TL, Gu F, Root S, Peck RM, Pan G, Yu J, Antosiewicz-Bourget J, Tian S, Stewart R, et al. 2008. NANOG is a direct target of TGF $\beta /$ activin-mediated SMAD signaling in human ESCs. Cell Stem Cell 3: 196-206.

Zafarana G, Avery SR, Avery K, Moore HD, Andrews PW. 2009. Specific knockdown of OCT4 in human embryonic stem cells by inducible short hairpin RNA interference. Stem Cells 27: 776-782.

Zhu Q, Luo K. 2012. SnoN in regulation of embryonic development and tissue morphogenesis. FEBS Lett 586: 1971-1976.

Zhu Q, Pearson-White S, Luo K. 2005. Requirement for the SnoN oncoprotein in transforming growth factor $\beta$-induced oncogenic transformation of fibroblast cells. Mol Cell Biol 25: 10731-10744.

Zhu Q, Krakowski AR, Dunham EE, Wang L, Bandyopadhyay A, Berdeaux R, Martin GS, Sun L, Luo K. 2007. Dual role of SnoN in mammalian tumorigenesis. Mol Cell Biol 27: 324-339. 


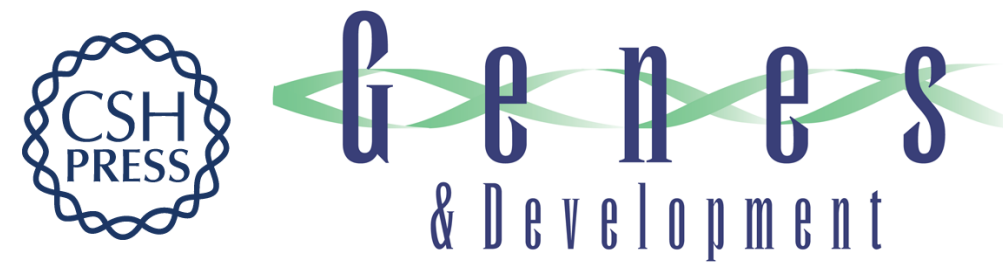

\section{The SMAD2/3 corepressor SNON maintains pluripotency through selective repression of mesendodermal genes in human ES cells}

Norihiro Tsuneyoshi, Ee Kim Tan, Akila Sadasivam, et al.

Genes Dev. 2012, 26:

Access the most recent version at doi:10.1101/gad.201772.112

Supplemental http://genesdev.cshlp.org/content/suppl/2012/11/12/26.22.2471.DC1
Material

References This article cites 33 articles, 8 of which can be accessed free at:

http://genesdev.cshlp.org/content/26/22/2471.full.html\#ref-list-1

License

Email Alerting Receive free email alerts when new articles cite this article - sign up in the box at the top

Service right corner of the article or click here.

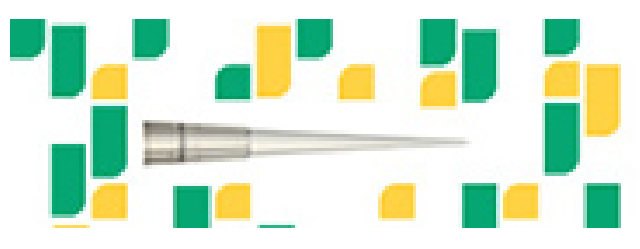

Focused on your science. 